\title{
A note on generalized bent criteria for Boolean functions
}

\author{
Sugata Gangopadhyay ${ }^{1}$, Enes Pasalic ${ }^{2}$, Pantelimon Stănică ${ }^{3}$ \\ ${ }^{1}$ Computer Science Unit \\ Indian Statistical Institute, Chennai Centre \\ Chennai - 600113, INDIA \\ sugodisichennai.res.in \\ ${ }^{2}$ University of Primorska, FAMNIT \\ Koper, SLOVENIA \\ enes.pasalic6@gmail.com \\ ${ }^{3}$ Department of Applied Mathematics \\ Naval Postgraduate School \\ Monterey, CA 93943-5216, USA \\ pstanica@nps.edu
}

\begin{abstract}
In this paper, we consider the spectra of Boolean functions with respect to the action of unitary transforms obtained by taking tensor products of the Hadamard kernel, denoted by $H$, and the negaHadamard kernel, denoted by $N$. The set of all such transforms is denoted by $\{H, N\}^{n}$. A Boolean function is said to be bent ${ }_{4}$ if its spectrum with respect to at least one unitary transform in $\{H, N\}^{n}$ is flat. We prove that the maximum possible algebraic degree of a bent ${ }_{4}$ function on $n$ variables is $\left\lceil\frac{n}{2}\right\rceil$, and hence solve an open problem posed by Riera and Parker [cf. IEEE-IT: 52:9 (2006), 4142-4159]. We obtain a relationship between bent and bent 4 functions which is a generalization of the relationship between bent and negabent Boolean functions proved by Parker and Pott [cf. LNCS: 4893 (2007), 9-23].
\end{abstract}

Keywords: Walsh-Hadamard transform, negaHadamard transform, bent function, bent 4 function, algebraic degree.

\section{INTRODUCTION}

Let us denote the set of integers, real numbers and complex numbers by $\mathbb{Z}, \mathbb{R}$ and $\mathbb{C}$, respectively and let the ring of integers modulo $r$ be denoted by $\mathbb{Z}_{r}$. The vector space $\mathbb{Z}_{2}^{n}$ is the space of all $n$-tuples $\mathbf{x}=$ $\left(x_{n}, \ldots, x_{1}\right)$ of elements from $\mathbb{Z}_{2}$ with the standard operations. By ' + ' we denote the addition over $\mathbb{Z}, \mathbb{R}$ and $\mathbb{C}$, whereas ' $\oplus$ ' denotes the addition over $\mathbb{Z}_{2}^{n}$ for all $n \geq 1$. Addition modulo $q$ is denoted by ' + ' and it is understood from the context. If $\mathbf{x}=\left(x_{n}, \ldots, x_{1}\right)$ and $\mathbf{y}=\left(y_{n}, \ldots, y_{1}\right)$ are in $\mathbb{Z}_{2}^{n}$, we define the scalar (or inner) product by $\mathbf{x} \cdot \mathbf{y}=x_{n} y_{n} \oplus \cdots \oplus x_{2} y_{2} \oplus x_{1} y_{1}$. The cardinality of a set $S$ is denoted by $|S|$. If $z=a+b \imath \in$
$\mathbb{C}$, then $|z|=\sqrt{a^{2}+b^{2}}$ denotes the absolute value of $z$, and $\bar{z}=a-b \imath$ denotes the complex conjugate of $z$, where $\imath^{2}=-1$, and $a, b \in \mathbb{R}$.

We call any function from $\mathbb{Z}_{2}^{n}$ to $\mathbb{Z}_{2}$ a Boolean function on $n$ variables and denote the set of all Boolean functions by $\mathcal{B}_{n}$. In general any function from $\mathbb{Z}_{2}^{n}$ to $\mathbb{Z}_{q}(q \geq 2$ a positive integer) is said to be a generalized Boolean function on $n$ variables [5], the set of all such functions being denoted by $\mathcal{G} \mathcal{B}_{n}^{q}$. Clearly $\mathcal{G B}_{n}^{2}=\mathcal{B}_{n}$. For any $f \in \mathcal{B}_{n}$, the algebraic normal form (ANF) is

$$
f\left(x_{n}, \ldots, x_{1}\right)=\bigoplus_{\mathbf{a}=\left(a_{n}, \ldots, a_{1}\right) \in \mathbb{Z}_{2}^{n}} \mu_{\mathbf{a}}\left(\prod_{i=1}^{n} x_{i}^{a_{i}}\right)
$$

where $\mu_{\mathbf{a}} \in \mathbb{Z}_{2}$, for all $\mathbf{a} \in \mathbb{Z}_{2}^{n}$. For any a $\in \mathbb{Z}_{2}^{n}$, $w t(\mathbf{a}):=\sum_{i=1}^{n} a_{i}$ is the Hamming weight. The algebraic degree of $f, \operatorname{deg}(f):=\max \{w t(\mathbf{a}): \mathbf{a} \in$ $\left.\mathbb{Z}_{2}^{n}, \mu_{\mathbf{a}} \neq 0\right\}$.

Now, let $q \geq 2$ be an integer, and let $\zeta=e^{2 \pi \imath / q}$ be the complex $q$-primitive root of unity. The WalshHadamard transform of $f \in \mathcal{G B}_{n}^{q}$ at any point $\mathbf{u} \in \mathbb{Z}_{2}^{n}$ is the complex valued function

$$
\mathcal{H}_{f}(\mathbf{u})=2^{-\frac{n}{2}} \sum_{\mathbf{x} \in \mathbb{Z}_{2}^{n}} \zeta^{f(\mathbf{x})}(-1)^{\mathbf{u} \cdot \mathbf{x}} .
$$

The inverse of the Walsh-Hadamard transform is given by

$$
\zeta^{f(\mathbf{y})}=2^{-\frac{n}{2}} \sum_{\mathbf{u} \in \mathbb{Z}_{2}^{n}} \mathcal{H}_{f}(\mathbf{u})(-1)^{\mathbf{u} \cdot \mathbf{y}} .
$$


A function $f \in \mathcal{G B}_{n}^{q}$ is a generalized bent function if and only if $\left|\mathcal{H}_{f}(\mathbf{u})\right|=1$ for all $\mathbf{u} \in \mathbb{Z}_{2}^{n}$. If $q=2$ and $n$ is even, then a generalized bent function is called a bent function. A function $f \in \mathcal{B}_{n}$, where $n$ is odd, is said to be semi-bent if and only if $\left|\mathcal{H}_{f}(\mathbf{u})\right| \in\{0, \sqrt{2}\}$, for all $\mathbf{u} \in \mathbb{Z}_{2}^{n}$. The maximum possible algebraic degree of a bent function on $n$ variables (when $n$ even) is $\frac{n}{2}$ and for a semi-bent function on $n$ variables (when $n$ odd) is $\frac{n+1}{2}$ (cf. [1], [2]).

The nega-Hadamard transform of $f \in \mathcal{B}_{n}$ at any vector $\mathbf{u} \in \mathbb{Z}_{2}^{n}$ is the complex valued function

$$
\mathcal{N}_{f}(\mathbf{u})=2^{-\frac{n}{2}} \sum_{\mathbf{x} \in \mathbb{Z}_{2}^{n}}(-1)^{f(\mathbf{x}) \oplus \mathbf{u} \cdot \mathbf{x}} \imath^{w t(\mathbf{x})} .
$$

A function $f \in \mathcal{B}_{n}$ is said to be negabent if and only if $\left|\mathcal{N}_{f}(\mathbf{u})\right|=1$ for all $\mathbf{u} \in \mathbb{Z}_{2}^{n}$. If $f \in \mathcal{B}_{n}$, then the inverse of the nega-Hadamard transform $\mathcal{N}_{f}$ is

$$
(-1)^{f(\mathbf{y})}=2^{-\frac{n}{2}} \imath^{-w t(\mathbf{y})} \sum_{\mathbf{u} \in \mathbb{Z}_{2}^{n}} \mathcal{N}_{f}(\mathbf{u})(-1)^{\mathbf{y} \cdot \mathbf{u}},
$$

for all $\mathbf{y} \in \mathbb{Z}_{2}^{n}$. We recall the following result.

Proposition 1: [6, Lemma 1] For any $\mathbf{u} \in \mathbb{Z}_{2}^{n}$ we have

$$
\sum_{\mathbf{x} \in \mathbb{Z}_{2}^{n}}(-1)^{\mathbf{u} \cdot \mathbf{x}} \imath^{w t(\mathbf{x})}=2^{\frac{n}{2}} \omega^{n} \imath^{-w t(\mathbf{u})},
$$

where $\omega=(1+\imath) / \sqrt{2}$ is a primitive 8 th root of unity.

The Hadamard kernel, the nega-Hadamard kernel and the identity transform on $\mathbb{Z}_{2}^{2}$, denoted by $H, N$ and $I$, respectively, are

$$
H=\frac{1}{\sqrt{2}}\left(\begin{array}{rr}
1 & 1 \\
1 & -1
\end{array}\right), N=\frac{1}{\sqrt{2}}\left(\begin{array}{rr}
1 & \imath \\
1 & -\imath
\end{array}\right)
$$

and

$$
I=\left(\begin{array}{ll}
1 & 0 \\
0 & 1
\end{array}\right)
$$

The set of $2^{n}$ different unitary transforms that are obtained by performing tensor products $H$ and $N, n$ times in any possible sequence is denoted by $\{H, N\}^{n}$. If $\mathbf{R}_{H}$ and $\mathbf{R}_{N}$ partition $\{1, \ldots, n\}$, then the unitary transform, $U$ of dimension $2^{n} \times 2^{n}$, corresponding to this partition is

$$
U=\prod_{j \in \mathbf{R}_{H}} H_{j} \prod_{j \in \mathbf{R}_{N}} N_{j}
$$

where

$$
K_{j}=I \otimes I \otimes \ldots \otimes I \otimes K \otimes I \otimes \ldots \otimes I
$$

with $K$ in the $j$ th position, $K \in\{H, N\}$ and " $\otimes$ " indicating the tensor product of matrices. Let $i_{\mathbf{x}} \in$ $\left\{0,1, \ldots, 2^{n}-1\right\}$ denote a row or column number of the unitary matrix $U$. We write

$$
i_{\mathbf{x}}=x_{n} 2^{n-1}+x_{n-1} 2^{n-2}+\cdots+x_{2} 2+x_{1}
$$

where $\mathbf{x}=\left(x_{n}, \ldots, x_{1}\right) \in \mathbb{Z}_{2}^{n}$. For any Boolean function $f \in \mathcal{B}_{n}$, let $(-1)^{\mathbf{f}}$ denote a $2^{n} \times 1$ column vector whose $i_{\mathbf{u}}$ row entry is $(-1)^{f(\mathbf{u})}$, for all $\mathbf{u} \in \mathbb{Z}_{2}^{n}$. The spectrum of $f$ with respect to $U \in\{H, N\}^{n}$ is the vector $U(-1)^{\mathbf{f}}$. If $\mathbf{R}_{H}=\{1, \ldots, n\}$, then the entry in the $i_{\mathbf{u}}$ th row of $U(-1)^{\mathbf{f}}$ is $\mathcal{H}_{f}(\mathbf{u})$ and, if $\mathbf{R}_{N}=\{1, \ldots, n\}$, then the entry in the $i_{\mathbf{u}}$ th row of $U(-1)^{\mathbf{f}}$ is $\mathcal{N}_{f}(\mathbf{u})$, for all $\mathbf{u} \in \mathbb{Z}_{2}^{n}$. In the former case, $U(-1)^{\mathbf{f}}$ is said to be the Walsh-Hadamard spectrum of $f$, while in the latter case it is the nega-Hadamard spectrum of $f$. The spectrum of a function $f$ with respect to a unitary transform $U$ is said to be flat if and only if the absolute value of each entry of $U(-1)^{\mathbf{f}}$ is 1 .

Definition 2: A function $f \in \mathcal{B}_{n}$ is said to be bent 4 if there exists at least one $U \in\{H, N\}^{n}$ such that $U(-1)^{\mathbf{f}}$ is flat.

The bent and the negabent functions belong to the class of bent 4 functions as extreme cases. For results on negabent and bent-negabent functions we refer to [3], [6], [7], [9].

In this paper, we consider the spectra of Boolean functions with respect to the action of unitary transforms in $\{H, N\}^{n}$. We prove that the maximum possible algebraic degree of a bent 4 function on $n$ variables is $\left\lceil\frac{n}{2}\right\rceil$, and hence solve an open problem posed by Riera and Parker [4]. Further, we obtain a

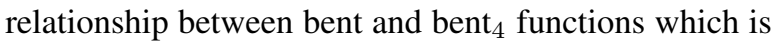
a generalization of the relationship between bent and negabent Boolean functions proved by Parker and Pott [3, Theorem 12].

\section{BENT PROPERTIES WITH RESPECT TO $\{H, N\}^{n}$}

Let $s_{r}(\mathbf{x})$ be the homogeneous symmetric Boolean function of algebraic degree $r$ whose ANF is

$$
s_{r}(\mathbf{x})=\bigoplus_{1 \leq i_{1}<\ldots<i_{r} \leq n} x_{i_{1}} \ldots x_{i_{r}} .
$$

The intersection of two vectors $\mathbf{c}=\left(c_{n}, \ldots, c_{1}\right), \mathbf{x}=$ $\left(x_{n}, \ldots, x_{1}\right) \in \mathbb{Z}_{2}^{n}$ is defined as

$$
\mathbf{c} * \mathbf{x}=\left(c_{n} x_{n}, \ldots, c_{1} x_{1}\right) .
$$

We define the function $s_{r}(\mathbf{c} * \mathbf{x})$ as

$$
s_{r}(\mathbf{c} * \mathbf{x})=\bigoplus_{1 \leq i_{1}<\ldots<i_{r} \leq n}\left(c_{i_{1}} x_{i_{1}}\right) \ldots\left(c_{i_{r}} x_{i_{r}}\right) .
$$

Suppose, the function $g \in \mathcal{G} \mathcal{B}_{n}^{4}$ defined as $g(\mathbf{x})=$ $w t(\mathbf{x}) \bmod 4$, for all $\mathbf{x} \in \mathbb{Z}_{2}^{n}$. In the following proposition and its corollary we obtain a connection between $g$ and $s_{2}$ which plays a crucial role in developing connections between different bent criteria. It is to be noted that the result of Proposition 3 is mentioned 
earlier by Su, Pott and Tang in the proof of [9, Lemma 1]. We provide an alternative proof.

Proposition 3: If $g \in \mathcal{G} \mathcal{B}_{n}^{4}$ is defined by $g(\mathbf{x})=$ $w t(\mathbf{x}) \bmod 4$ for all $\mathbf{x} \in \mathbb{Z}_{2}^{n}$, then

$$
g(\mathbf{x})=\mathbf{1} \cdot \mathbf{x}+2 s_{2}(\mathbf{x})=w t(\mathbf{x}) \bmod 4,
$$

for all $\mathrm{x} \in \mathbb{Z}_{2}^{n}$.

Proof: By Proposition 1, we have

$$
2^{-\frac{n}{2}} \sum_{\mathbf{x} \in \mathbb{Z}_{2}^{n}}(-1)^{\mathbf{u} \cdot \mathbf{x}} \imath^{w t(\mathbf{x})}=\omega^{n} \imath^{-w t(\mathbf{u})} .
$$

Therefore, $g(\mathbf{x})=w t(\mathbf{x}) \bmod 4$ is a generalized bent on $\mathbb{Z}_{4}$, which we refer to as $\mathbb{Z}_{4}$-bent. According to [8, Corollary 15] and [5], there exist $a, b \in \mathcal{B}_{n}$ such that $b$ and $a+b$ are bent functions and $g(\mathbf{x})=a(\mathbf{x})+2 b(\mathbf{x})=$ $w t(\mathbf{x}) \bmod 4$, for all $\mathbf{x} \in \mathbb{Z}_{2}^{n}$. From this we have

$$
2 b(\mathbf{x}) \equiv w t(\mathbf{x})-a(\mathbf{x}) \quad(\bmod 4)
$$

i.e.,

$$
2 \mid(w t(\mathbf{x})-a(\mathbf{x})),
$$

i.e.,

$$
a(\mathbf{x})=\mathbf{1} \cdot \mathbf{x}
$$

where $\mathbf{1}=(1,1, \ldots, 1) \in \mathbb{Z}_{2}^{n}$, for all $\mathbf{x} \in \mathbb{Z}_{2}^{n}$. Therefore,

$g(\mathbf{x})=\mathbf{1} \cdot \mathbf{x}+2 b(\mathbf{x})=w t(\mathbf{x}) \quad \bmod 4$, for all $\mathbf{x} \in \mathbb{Z}_{2}^{n}$,

i.e.,

$$
b(\mathbf{x})=\frac{-\mathbf{1} \cdot \mathbf{x}+w t(\mathbf{x})}{2} \quad \bmod 2, \text { for all } \mathbf{x} \in \mathbb{Z}_{2}^{n} .
$$

Since $b \in \mathcal{B}_{n}$ is a symmetric bent function and $b(\mathbf{0})=0$ we have $b(\mathbf{x})=s_{2}(\mathbf{x})$ or $s_{2}(\mathbf{x}) \oplus s_{1}(\mathbf{x})$. Since $b(0 \ldots 01)=0$, we have $b(\mathbf{x})=s_{2}(\mathbf{x})$. Therefore

$g(\mathbf{x})=\mathbf{1} \cdot \mathbf{x}+2 s_{2}(\mathbf{x})=w t(\mathbf{x}) \quad \bmod 4$, for all $\mathbf{x} \in \mathbb{Z}_{2}^{n}$.

The following corollary generalizes (10) which is useful in finding a general expression of entries of any matrix $U \in\{H, N\}^{n}$.

Corollary 4: Let $\mathbf{x}, \mathbf{c} \in \mathbb{Z}_{2}^{n}$. Then

$$
\mathbf{c} \cdot \mathbf{x}+2 s_{2}(\mathbf{c} * \mathbf{x})=w t(\mathbf{c} * \mathbf{x}) \quad \bmod 4,
$$

for all $\mathrm{x} \in \mathbb{Z}_{2}^{n}$.

Proof: In Proposition 3 it is proved that

$\mathbf{1} \cdot \mathbf{x}+2 s_{2}(\mathbf{x})=w t(\mathbf{x}) \quad \bmod 4$, for all $\mathbf{x} \in \mathbb{Z}_{2}^{n}$, i.e.,

$$
\begin{gathered}
(1, \ldots, 1) \cdot\left(x_{n}, \ldots, x_{1}\right)+2 s_{2}\left(x_{n}, \ldots, x_{1}\right) \\
=w t\left(x_{n}, \ldots, x_{1}\right) \quad \bmod 4, \text { for all } \mathbf{x} \in \mathbb{Z}_{2}^{n} .
\end{gathered}
$$

Replacing $x_{i}$ by $c_{i} x_{i}$ we get

$$
\begin{gathered}
(1, \ldots, 1) \cdot\left(c_{n} x_{n}, \ldots, c_{1} x_{1}\right)+2 s_{2}\left(c_{n} x_{n}, \ldots, c_{1} x_{1}\right) \\
\quad=w t\left(c_{n} x_{n}, \ldots, c_{1} x_{1}\right) \quad \bmod 4, \text { for all } \mathbf{x} \in \mathbb{Z}_{2}^{n},
\end{gathered}
$$

i.e.,

$$
\left(c_{n} x_{n} \oplus \ldots \oplus c_{1} x_{1}\right)+2 s_{2}\left(c_{n} x_{n}, \ldots, c_{1} x_{1}\right)
$$
$=w t\left(c_{n} x_{n}, \ldots, c_{1} x_{1}\right) \quad \bmod 4$, for all $\mathbf{x} \in \mathbb{Z}_{2}^{n}$.

Therefore,

$\mathbf{c} \cdot \mathbf{x}+2 s_{2}(\mathbf{c} * \mathbf{x})=w t(\mathbf{c} * \mathbf{x}) \quad \bmod 4$, for all $\mathbf{x} \in \mathbb{Z}_{2}^{n}$.

Riera and Parker [4, Lemma 7] have obtained a general expression for the entries of any matrix $U \in\{H, N\}^{n}$. We obtain an alternative description below which we use to connect the spectrum $U(-1)^{\mathbf{f}}$ of any $f \in \mathcal{B}_{n}$ to the Walsh-Hadamard spectra of some associated functions.

Theorem 5: If $U=\prod_{j \in \mathbf{R}_{H}} H_{j} \prod_{j \in \mathbf{R}_{N}} N_{j}$, is a unitary matrix constructed as in (7), corresponding to the partition $\mathbf{R}_{H}, \mathbf{R}_{N}$ of $\{1, \ldots, n\}$ where $n \geq 2$, then for any $\mathbf{u}, \mathbf{x} \in \mathbb{Z}_{2}^{n}$ the entry in the $i_{\mathbf{u}}$ th row and $i_{\mathbf{x}}$ th column of $2^{\frac{n}{2}} U$ is

$$
(-1)^{\mathbf{u} \cdot \mathbf{x} \oplus s_{2}(\mathbf{c} * \mathbf{x})} \imath^{\mathbf{c} \cdot \mathbf{x}},
$$

where $\mathbf{c}=\left(c_{n}, \ldots, c_{1}\right) \in \mathbb{Z}_{2}^{n}$ is such that $c_{i}=0$ if $i \in \mathbf{R}_{H}$ and $c_{i}=1$ if $i \in \mathbf{R}_{N}$.

Proof: We prove this by induction. Let $n=2$. If $\mathbf{c}=(0,0)$ then clearly $U=H \otimes H$, and if $\mathbf{c}=(1,1)$ then $U=N \otimes N$. We explicitly compute $U$ when $\mathbf{c}=(0,1)$ and $\mathbf{c}=(1,0)$ and find that $U$ is equal to

$$
H \otimes N=\frac{1}{2}\left(\begin{array}{rrrr}
1 & \imath & 1 & \imath \\
1 & -\imath & 1 & -\imath \\
1 & \imath & -1 & -\imath \\
1 & -\imath & -1 & \imath
\end{array}\right),
$$

and

$$
N \otimes H=\frac{1}{2}\left(\begin{array}{rrrr}
1 & 1 & \imath & \imath \\
1 & -1 & \imath & -\imath \\
1 & 1 & -\imath & -\imath \\
1 & -1 & -\imath & \imath
\end{array}\right),
$$

respectively. By Corollary 4

$$
(-1)^{\mathbf{u} \cdot \mathbf{x} \oplus s_{2}(\mathbf{c} * \mathbf{x})} \imath^{\mathbf{c} \cdot \mathbf{x}}=(-1)^{\mathbf{u} \cdot \mathbf{x}} \imath^{w t(\mathbf{c} * \mathbf{x})} .
$$

Suppose the result is true for $n$. Let $\mathbf{u}, \mathbf{x}, \mathbf{c} \in \mathbb{Z}_{2}^{n}$, and $\mathbf{u}^{\prime}=\left(u_{n+1}, \mathbf{u}\right), \mathbf{x}^{\prime}=\left(x_{n+1}, \mathbf{x}\right), \mathbf{c}^{\prime}=\left(c_{n+1}, \mathbf{c}\right) \in$ $\mathbb{Z}_{2}^{n+1}$. Let $U \in\{H, N\}^{n}$ be the unitary transform induced by the partition corresponding to $\mathbf{c} \in \mathbb{Z}_{2}^{n}$. The transform corresponding to the partition induced by 
$\mathbf{c}^{\prime}=(0, \mathbf{c}) \in \mathbb{Z}_{2}^{n+1}$ is $H \otimes U$. By taking the tensor Therefore, product of $H$ and $U$ we obtain

$$
2^{\frac{n+1}{2}}(H \otimes U)=\left(\begin{array}{ll}
A_{11} & A_{12} \\
A_{21} & A_{22}
\end{array}\right)
$$

$$
2^{\frac{n+1}{2}}(N \otimes U)=\left((-1)^{\mathbf{u}^{\prime} \cdot \mathbf{x}^{\prime}} \imath^{w t\left(\mathbf{c}^{\prime} * \mathbf{x}^{\prime}\right)}\right)_{2^{n+1} \times 2^{n+1}} .
$$

where

$$
\begin{aligned}
A_{11} & =\left((-1)^{\mathbf{u} \cdot \mathbf{x}} \imath^{w t(\mathbf{c} * \mathbf{x})}\right)_{2^{n} \times 2^{n}} \\
& =\left((-1)^{(0, \mathbf{u}) \cdot(0, \mathbf{x})} \imath^{w t((0, \mathbf{c}) *(0, \mathbf{x}))}\right)_{2^{n} \times 2^{n}}, \\
A_{12} & =\left((-1)^{\mathbf{u} \cdot \mathbf{x}} \imath^{w t(\mathbf{c} * \mathbf{x})}\right)_{2^{n} \times 2^{n}} \\
& =\left((-1)^{(0, \mathbf{u}) \cdot(1, \mathbf{x})} \imath^{w t((0, \mathbf{c}) *(1, \mathbf{x}))}\right)_{2^{n} \times 2^{n}}, \\
A_{21} & =\left((-1)^{\mathbf{u} \cdot \mathbf{x}} \imath^{w t(\mathbf{c} * \mathbf{x})}\right)_{2^{n} \times 2^{n}} \\
& =\left((-1)^{(1, \mathbf{u}) \cdot(0, \mathbf{x})} \imath^{w t((0, \mathbf{c}) *(0, \mathbf{x}))}\right)_{2^{n} \times 2^{n}}
\end{aligned}
$$

and

$$
\begin{aligned}
A_{22} & =\left((-1)(-1)^{\mathbf{u} \cdot \mathbf{x}} \imath^{w t(\mathbf{c} * \mathbf{x})}\right)_{2^{n} \times 2^{n}} \\
& =\left((-1)^{(1, \mathbf{u}) \cdot(1, \mathbf{x})} \imath^{w t((0, \mathbf{c}) *(1, \mathbf{x}))}\right)_{2^{n} \times 2^{n}} .
\end{aligned}
$$

Therefore,

$$
2^{\frac{n+1}{2}}(H \otimes U)=\left((-1)^{\mathbf{u}^{\prime} \cdot \mathbf{x}^{\prime}} \imath^{w t\left(\mathbf{c}^{\prime} * \mathbf{x}^{\prime}\right)}\right)_{2^{n+1} \times 2^{n+1}} .
$$

The transform corresponding to the partition induced by $\mathbf{c}^{\prime}=(1, \mathbf{c}) \in \mathbb{Z}_{2}^{n+1}$ is $N \otimes U$. By taking the tensor product of $H$ and $U$ we obtain

$$
2^{\frac{n+1}{2}}(N \otimes U)=\left(\begin{array}{cc}
B_{11} & B_{12} \\
B_{21} & B_{22}
\end{array}\right)
$$

where

$$
\begin{aligned}
B_{11} & =\left((-1)^{\mathbf{u} \cdot \mathbf{x}} \imath^{w t(\mathbf{c} * \mathbf{x})}\right)_{2^{n} \times 2^{n}} \\
& =\left((-1)^{(0, \mathbf{u}) \cdot(0, \mathbf{x})} \imath^{w t((1, \mathbf{c}) *(0, \mathbf{x}))}\right)_{2^{n} \times 2^{n}}, \\
B_{12} & =\left(\imath(-1)^{\mathbf{u} \cdot \mathbf{x}} \imath^{w t(\mathbf{c} * \mathbf{x})}\right)_{2^{n} \times 2^{n}} \\
& =\left((-1)^{(0, \mathbf{u}) \cdot(1, \mathbf{x})} \imath^{w t((1, \mathbf{c}) *(1, \mathbf{x}))}\right)_{2^{n} \times 2^{n}}, \\
B_{21} & =\left((-1)^{\mathbf{u} \cdot \mathbf{x}} \imath^{w t(\mathbf{c} * \mathbf{x})}\right)_{2^{n} \times 2^{n}} \\
& =\left((-1)^{(1, \mathbf{u}) \cdot(0, \mathbf{x})} \imath^{w t((1, \mathbf{c}) *(0, \mathbf{x}))}\right)_{2^{n} \times 2^{n}}
\end{aligned}
$$

and

$$
\begin{aligned}
B_{22} & =\left((-\imath)(-1)^{\mathbf{u} \cdot \mathbf{x}} \imath^{w t(\mathbf{c} * \mathbf{x})}\right)_{2^{n} \times 2^{n}} \\
& =\left((-1)^{(1, \mathbf{u}) \cdot(1, \mathbf{x})} \imath^{w t((1, \mathbf{c}) *(1, \mathbf{x}))}\right)_{2^{n} \times 2^{n}} .
\end{aligned}
$$

This proves the result.

Using Theorem 5 we can state that given any $U \in$ $\{H, N\}^{n}$ there exists $\mathbf{c} \in \mathbb{Z}_{2}^{n}$ such that for any $f \in \mathcal{B}_{n}$ the $i_{\mathbf{u}}$ th row of the column vector $U(-1)^{\mathbf{f}}$ is

$$
\begin{aligned}
\mathcal{U}_{f}^{\mathbf{c}}(\mathbf{u})= & 2^{-\frac{n}{2}} \sum_{\mathbf{x} \in \mathbb{Z}_{2}^{n}}(-1)^{f(\mathbf{x}) \oplus s_{2}(\mathbf{c} * \mathbf{x})} \imath^{\mathbf{c} \cdot \mathbf{x}}(-1)^{\mathbf{u} \cdot \mathbf{x}} \\
= & 2^{-\frac{n}{2}} \sum_{\mathbf{x} \in \mathbf{c}^{\perp}}(-1)^{f(\mathbf{x}) \oplus s_{2}(\mathbf{c} * \mathbf{x})}(-1)^{\mathbf{u} \cdot \mathbf{x}} \\
& +\imath 2^{-\frac{n}{2}} \sum_{\mathbf{x} \notin \mathbf{c}^{\perp}}(-1)^{f(\mathbf{x}) \oplus s_{2}(\mathbf{c} * \mathbf{x})}(-1)^{\mathbf{u} \cdot \mathbf{x}} .
\end{aligned}
$$

Therefore, $\mathcal{U}_{f}^{\mathbf{c}}(\mathbf{u})$ is related to the Walsh-Hadamard transform of restrictions $f(\mathbf{x}) \oplus s_{2}(\mathbf{c} * \mathbf{x})$ to the subspace $\mathbf{c}^{\perp}$ and its coset. From another perspective this transform provides a measure of the distance of the function $f$ to the functions of the form $s_{2}(\mathbf{c} * \mathbf{x}) \oplus \mathbf{u} \cdot \mathbf{x}$. Thus, if $\left|\mathcal{U}_{f}^{\mathbf{c}}(\mathbf{u})\right|$ has high value for a choice of $\mathbf{u}, \mathbf{c} \in \mathbb{Z}_{2}^{n}$ then $f$ has low Hamming distance from the function of the form $s_{2}(\mathbf{c} * \mathbf{x}) \oplus \mathbf{u} \cdot \mathbf{x}$. This means that the function may be approximated efficiently by the function $s_{2}(\mathbf{c} * \mathbf{x}) \oplus \mathbf{u} \cdot \mathbf{x}$. This may have some cryptographic significance for the spectra of $f$ with respect to the transform $U \in\{H, N\}^{n}$.

Riera and Parker [4, p. 4125 ] posed the following open problem:

What is the maximum algebraic degree of a bent $_{4}$ Boolean function of $n$ variables?

Su, Pott and Tang [9] have recently proved that the maximum algebraic degree of a bent-negabent function is $\frac{n}{2}$ (note that $n$ is even, since bent functions exist only on even variables). Further, they have provided a method to construct bent-negabent functions of algebraic degree ranging from 2 to $\frac{n}{2}$. In the next theorem we solve the problem proposed by Riera and Parker and thus generalize the result related to the upper bound of algebraic degree proved in [9].

Theorem 6: The maximum algebraic degree of a bent $_{4}$ Boolean function on $n$ variables is $\left\lceil\frac{n}{2}\right\rceil$.

Proof: Using Theorem 5 we can state that given any $U \in\{H, N\}^{n}$ there exists $\mathbf{c} \in \mathbb{Z}_{2}^{n}$ such that for any $f \in \mathcal{B}_{n}$ the $i_{\mathbf{u}}$ th row entry of the column vector 


$$
\begin{aligned}
& U(-1)^{\mathbf{f}} \text { is } \\
& 2^{\frac{n}{2}} \mathcal{U}_{f}^{\mathbf{c}}(\mathbf{u})=\sum_{\mathbf{x} \in \mathbb{Z}_{2}^{n}}(-1)^{f(\mathbf{x}) \oplus s_{2}(\mathbf{c} * \mathbf{x})} \imath^{\mathbf{c} \cdot \mathbf{x}}(-1)^{\mathbf{u} \cdot \mathbf{x}} \\
& =\sum_{\mathbf{x} \in \mathbf{c}^{\perp}}(-1)^{f(\mathbf{x}) \oplus s_{2}(\mathbf{c} * \mathbf{x})}(-1)^{\mathbf{u} \cdot \mathbf{x}} \\
& +\imath \sum_{\mathbf{x} \notin \mathbf{c}^{\perp}}(-1)^{f(\mathbf{x}) \oplus s_{2}(\mathbf{c} * \mathbf{x})}(-1)^{\mathbf{u} \cdot \mathbf{x}} .
\end{aligned}
$$

Let us suppose that $f$ is bent $_{4}$ with respect to the chosen transform $U$. Therefore, we have $\left|\mathcal{U}_{f}^{\mathbf{c}}(\mathbf{u})\right|=1$, for all $\mathbf{u} \in \mathbb{Z}_{2}^{n}$. By (14)

$$
\begin{aligned}
2^{n}= & \left(\sum_{\mathbf{x} \in \mathbf{c}^{\perp}}(-1)^{f(\mathbf{x}) \oplus s_{2}(\mathbf{c} * \mathbf{x})}(-1)^{\mathbf{u} \cdot \mathbf{x}}\right)^{2} \\
& +\left(\sum_{\mathbf{x} \notin \mathbf{c}^{\perp}}(-1)^{f(\mathbf{x}) \oplus s_{2}(\mathbf{c} * \mathbf{x})}(-1)^{\mathbf{u} \cdot \mathbf{x}}\right)^{2} .
\end{aligned}
$$

By Jacobi's two-square theorem we know that $2^{n}$ has a unique representation (disregarding the sign and order) as a sum of two squares, namely $2^{n}=\left(2^{\frac{n}{2}}\right)^{2}+0$, if $n$ is even, and $2^{n}=\left(2^{\frac{n-1}{2}}\right)^{2}+\left(2^{\frac{n-1}{2}}\right)^{2}$, if $n$ is odd. Let $g_{\mathbf{c}}(\mathbf{x})=s_{2}(\mathbf{c} * \mathbf{x})$, for all $\mathbf{x} \in \mathbb{Z}_{2}^{n}$.

$$
\begin{aligned}
\left|\mathcal{H}_{f \oplus g_{\mathbf{c}}}(\mathbf{u})\right|= & \left|2^{-\frac{n}{2}} \sum_{\mathbf{x} \in \mathbb{Z}_{2}^{n}}(-1)^{f(\mathbf{x}) \oplus s_{2}(\mathbf{c} * \mathbf{x})}(-1)^{\mathbf{u} \cdot \mathbf{x}}\right| \\
= & \mid 2^{-\frac{n}{2}} \sum_{\mathbf{x} \in \mathbf{c}^{\perp}}(-1)^{f(\mathbf{x}) \oplus s_{2}(\mathbf{c} * \mathbf{x})}(-1)^{\mathbf{u} \cdot \mathbf{x}} \\
& +2^{-\frac{n}{2}} \sum_{\mathbf{x} \notin \mathbf{c}^{\perp}}(-1)^{f(\mathbf{x}) \oplus s_{2}(\mathbf{c} * \mathbf{x})}(-1)^{\mathbf{u} \cdot \mathbf{x}} \mid \\
= & 1,
\end{aligned}
$$

for all $\mathbf{u} \in \mathbb{Z}_{2}^{n}$. Therefore, $f \oplus g_{\mathbf{c}}$ is a bent function and its algebraic degree is bounded above by $\frac{n}{2}$. The algebraic degree of $g_{\mathbf{c}}$ is upper-bounded by 2 , so the upper bound of the algebraic degree of a bent ${ }_{4}$ Boolean function $f$ is $\frac{n}{2}$, when $n$ is even.

In case $n$ is odd by a similar argument we get $\left|\mathcal{H}_{f \oplus g_{\mathbf{c}}}(\mathbf{u})\right| \in\{0, \sqrt{2}\}$, that is $f \oplus g_{\mathbf{c}}$ is semi-bent, and therefore the algebraic degree of $f$ is bounded above by $\frac{n+1}{2}$.

\section{CONNECTING BENT AND BENT 4 FUNCTIONS}

Let $f \in \mathcal{B}_{n}$ and $V$ is a subspace of $\mathbb{Z}_{2}^{n}$. For any $\mathbf{a} \in \mathbb{Z}_{2}^{n}$ the restriction of $f$ to the coset $\mathbf{a}+V$ is defined as $\left.f\right|_{\mathbf{a}+V}(\mathbf{x})=f(\mathbf{a}+\mathbf{x})$, for all $\mathbf{x} \in V$. It is to be noted that the restriction of a function $f$ to a coset $\mathbf{a}+V$ is unique up to a translation. The following lemma is well known (cf. [1]), nevertheless we provide a complete proof for clarity.
Lemma 7: Let $n=2 k, f \in \mathcal{B}_{n}$ a bent function, $V$ be an $(n-1)$-dimensional subspace of $\mathbb{Z}_{2}^{n}$, a $\in \mathbb{Z}_{2}^{n} \backslash V$ such that $\mathbb{Z}_{2}^{n}=V \cup(\mathbf{a} \oplus V)$. Then the restrictions of $f$ to $V$ and $\mathbf{a} \oplus V$, denoted $\left.f\right|_{V}$ and $\left.f\right|_{\mathbf{a} \oplus V}$ respectively, are semi-bent functions and $\mathcal{H}_{\left.f\right|_{V}}(\mathbf{u}) \mathcal{H}_{\left.f\right|_{\mathbf{a} \oplus V}}(\mathbf{u})=0$ for all $\mathbf{u} \in \mathbb{F}_{2}^{n}$.

Proof: Since the dimension of $V$ is $n-1$, the dimension of the orthogonal subspace $V^{\perp}$ is 1 . Let $V^{\perp}=\{\mathbf{0}, \mathbf{b}\}$. Since $\mathbf{a} \notin V, \mathbf{a} \cdot \mathbf{b}=1$. For all $\mathbf{u} \in \mathbb{Z}_{2}^{n}$ we have the following

$$
\begin{aligned}
2^{\frac{n}{2}} \mathcal{H}_{f}(\mathbf{u})= & \sum_{\mathbf{x} \in V}(-1)^{f(\mathbf{x}) \oplus \mathbf{u} \cdot \mathbf{x}} \\
& +(-1)^{\mathbf{u} \cdot \mathbf{a}} \sum_{\mathbf{x} \in V}(-1)^{f(\mathbf{x}+\mathbf{a}) \oplus \mathbf{u} \cdot \mathbf{x}} \\
& \in\left\{-2^{\frac{n}{2}}, 2^{\frac{n}{2}}\right\}
\end{aligned}
$$

$$
\begin{aligned}
2^{\frac{n}{2}} \mathcal{H}_{f}(\mathbf{u} \oplus \mathbf{b})= & \sum_{\mathbf{x} \in V}(-1)^{f(\mathbf{x}) \oplus \mathbf{u} \cdot \mathbf{x}} \\
& -(-1)^{\mathbf{u} \cdot \mathbf{a}} \sum_{\mathbf{x} \in V}(-1)^{f(\mathbf{x}+\mathbf{a}) \oplus \mathbf{u} \cdot \mathbf{x}} \\
& \in\left\{-2^{\frac{n}{2}}, 2^{\frac{n}{2}}\right\} .
\end{aligned}
$$

By adding (17) and (18) we obtain $\sum_{\mathbf{x} \in V}(-1)^{f(\mathbf{x}) \oplus \mathbf{u} \cdot \mathbf{x}} \in\left\{-2^{\frac{n}{2}}, 0,2^{\frac{n}{2}}\right\}, \quad$ and by subtracting (18) from (17) we obtain $\sum_{\mathbf{x} \in V}(-1)^{f(\mathbf{a} \oplus \mathbf{x}) \oplus \mathbf{u} \cdot \mathbf{x}} \in\left\{-2^{\frac{n}{2}}, 0,2^{\frac{n}{2}}\right\}$. This proves that both $\left.f\right|_{V}$ and $\left.f\right|_{\mathbf{a} \oplus V}$ are semi-bent functions. Further, since the sums in (17) and (18) are both in $\left\{-2^{\frac{n}{2}}, 2^{\frac{n}{2}}\right\}$, for all $\mathbf{u} \in \mathbb{Z}_{2}^{n}$, we have $\mathcal{H}_{\left.f\right|_{V}}(\mathbf{u}) \mathcal{H}_{\left.f\right|_{\mathbf{a} \oplus V}}(\mathbf{u})=0$, for all $\mathbf{u} \in \mathbb{Z}_{2}^{n}$.

This leads us to a generalization of [3, Theorem 12] due to Parker and Pott. Recall that for any $\mathbf{c} \in \mathbb{Z}_{2}^{n}$ we have defined $g_{\mathbf{c}}(\mathbf{x})=s_{2}(\mathbf{c} * \mathbf{x})$, for all $\mathbf{x} \in \mathbb{Z}_{2}^{n}$.

Theorem 8: Let $f \in \mathcal{B}_{n}$ where $n$ is even. Then the following two statements are true.

1) If $f$ is bent, then $f \oplus g_{\mathbf{c}}$ is bent 4 and $\left|\mathcal{U}_{f \oplus g_{\mathbf{c}}}^{\mathbf{c}}(\mathbf{u})\right|=1$, for all $\mathbf{u} \in \mathbb{Z}_{2}^{n}$

2) If $f$ is bent 4 , i.e., there exists $\mathbf{c} \in \mathbb{Z}_{2}^{n}$ such that $\left|\mathcal{U}_{f}^{\mathbf{c}}(\mathbf{u})\right|=1$, for all $\mathbf{u} \in \mathbb{Z}_{2}^{n}$, then $f \oplus g_{\mathbf{c}}$ is bent. 
Proof: Suppose $f$ is a bent function. If $\mathbf{c}=\mathbf{0}$ there is nothing to prove. If $\mathbf{c} \neq \mathbf{0}$, then

$$
\begin{aligned}
2^{\frac{n}{2}} \mathcal{U}_{f \oplus g_{\mathbf{c}}}^{\mathbf{c}}(\mathbf{u})= & \sum_{\mathbf{x} \in \mathbb{Z}_{2}^{n}}(-1)^{f(\mathbf{x}) \oplus s_{2}(\mathbf{c} * \mathbf{x})} \imath^{w t(\mathbf{c} * \mathbf{x})}(-1)^{\mathbf{u} \cdot \mathbf{x}} \\
= & \sum_{\mathbf{x} \in \mathbb{Z}_{2}^{n}}(-1)^{f(\mathbf{x}) \oplus s_{2}(\mathbf{c} * \mathbf{x}) \oplus s_{2}(\mathbf{c} * \mathbf{x}) \oplus \mathbf{u} \cdot \mathbf{x}} \imath^{\mathbf{c} \cdot \mathbf{x}} \\
= & \sum_{\mathbf{x} \in \mathbb{Z}_{2}^{n}}(-1)^{f(\mathbf{x}) \oplus \mathbf{u} \cdot \mathbf{x}} \imath^{\mathbf{c} \cdot \mathbf{x}} \\
= & \sum_{\mathbf{x} \in \mathbf{c}^{\perp}}(-1)^{f(\mathbf{x}) \oplus \mathbf{u} \cdot \mathbf{x}} \\
& +\imath \sum_{\mathbf{x} \notin \mathbf{c}^{\perp}}(-1)^{f(\mathbf{x}) \oplus \mathbf{u} \cdot \mathbf{x} .}
\end{aligned}
$$

Since $f$ is a bent function and $\mathbf{c}^{\perp}$ is a subspace of codimension 1 , by Lemma 7 the restrictions of $f$ on $\mathbf{c}^{\perp}$ and its remaining coset are semi-bent and their Walsh-Hadamard spectra are disjoint. Therefore, the right hand side of the above equation belongs to the set $\left\{ \pm 2^{\frac{n}{2}}, \pm 2^{\frac{n}{2}} \imath\right\}$ for all $\mathbf{u} \in \mathbb{Z}_{2}^{n}$. This proves the first statement.

In the second part, we assume $f$ to be a bent 4 function such that there exists $\mathbf{c} \in \mathbb{Z}_{2}^{n}$ for which $\left|\mathcal{U}_{f}^{\mathbf{c}}(\mathbf{u})\right|=1$, for all $\mathbf{u} \in \mathbb{Z}_{2}^{n}$. We have

$$
\begin{aligned}
\mathcal{U}_{f}^{\mathbf{c}}(\mathbf{u}) & =2^{-\frac{n}{2}} \sum_{\mathbf{x} \in \mathbb{Z}_{2}^{n}}(-1)^{f(\mathbf{x})} \imath^{w t(\mathbf{c} * \mathbf{x})}(-1)^{\mathbf{u} \cdot \mathbf{x}} \\
& =2^{-\frac{n}{2}} \sum_{\mathbf{x} \in \mathbb{Z}_{2}^{n}} \imath^{w t(\mathbf{c} * \mathbf{x})+2 f(\mathbf{x})}(-1)^{\mathbf{u} \cdot \mathbf{x}}
\end{aligned}
$$

Thus, the function $h(\mathbf{x})=w t(\mathbf{c} * \mathbf{x})+2 f(\mathbf{x}) \bmod 4$, for all $\mathbf{x} \in \mathbb{Z}_{2}^{n}$, is a $\mathbb{Z}_{4}$-bent function which implies the existence of Boolean functions $a, b \in \mathcal{B}_{n}$ such that $b, a+b$ are bents [8, Corollary 15], with

$$
\begin{aligned}
h(\mathbf{x}) & =a(\mathbf{x})+2 b(\mathbf{x}) \\
& =w t(\mathbf{c} * \mathbf{x})+2 f(\mathbf{x}) \quad \bmod 4,
\end{aligned}
$$

for all $\mathbf{x} \in \mathbb{Z}_{2}^{n}$. Therefore, $2 \mid(a(\mathbf{x})-w t(\mathbf{c} * \mathbf{x}))$, which implies $a(\mathbf{x})=\mathbf{c} \cdot \mathbf{x}$, for all $\mathbf{x} \in \mathbb{Z}_{2}^{n}$. By Corollary 4 and (20) we have

$$
b(\mathbf{x})=f(\mathbf{x}) \oplus s_{2}(\mathbf{c} * \mathbf{x}),
$$

for all $\mathbf{x} \in \mathbb{Z}_{2}^{n}$. Since $b \in \mathcal{B}_{n}$ is a bent function, $f \oplus g_{\mathbf{c}}$ is a bent function. Thus, we have proved that if $f$ is bent $_{4}$ function, with respect to the unitary transform corresponding to $\mathbf{c} \in \mathbb{Z}_{2}^{n}$, then $f \oplus g_{\mathbf{c}}$ is a bent function.

\section{CONCLUSION}

In this paper we have developed an approach to study the action of the transforms in $\{H, N\}^{n}$ on
Boolean functions on $n$ variables. By using our approach we have proved that the maximum possible algebraic degree of a bent 4 function on $n$ variables is $\left\lceil\frac{n}{2}\right\rceil$ and hence solve an open problem proposed by Riera and Parker [4]. We have also obtained a connection between bent and bent ${ }_{4}$ functions, which is a generalization of the connection between bent and negabent function proved by Parker and Pott [3]. It is observed that if the absolute value of an entry in the spectrum of a function with respect to a transform $U \in\{H, N\}^{n}$ is large, then the function has low Hamming distance from a particular quadratic function determined by $U$. Thus, we have established a connection between the generalized bent criteria and approximation of a Boolean function of arbitrary algebraic degree by quadratic Boolean functions.

\section{REFERENCES}

[1] C. Carlet, "Boolean functions for cryptography and error correcting codes," in Boolean Models and Methods in Mathematics, Computer Science, and Engineering, Y. Crama and P. L. Hammer, Eds. Cambridge, U.K.: Cambridge Univ. Press, 2010, pp. 257-397.

[2] T. W. Cusick, P. Stănică, Cryptographic Boolean functions and applications. New York: Academic, 2009.

[3] M. G. Parker, A. Pott, "On Boolean functions which are bent and negabent," in Proc. Int. Conf. Sequences, Subsequences, Consequences, 2007, vol. LNCS-4893, pp. 9-23.

[4] C. Riera, M. G. Parker, "Generalized bent criteria for Boolean functions," IEEE Trans. Inf. Theory, vol. 52, no. 9, pp. 41424159, Sep. 2006.

[5] P. Solé, N. Tokareva, "Connections between Quaternary and Binary Bent Functions," Prikl. Diskr. Mat., vol 1, pp. 16-18, 2009, (http://eprint.iacr.org/2009/544.pdf).

[6] K. U. Schmidt, M. G. Parker, A. Pott, "Negabent functions in the Maiorana-McFarland class," in Proc. Int. Conf. Sequences Appl., 2008, vol. LNCS-5203, pp. 390-402.

[7] P. Stănică, S. Gangopadhyay, A. Chaturvedi, A. K. Gangopadhyay, S. Maitra, "Investigations on bent and negabent functions via the nega-Hadamard transform," IEEE Trans. Inf. Theory, vol. 58, no. 6, pp. 4064-4072, June 2012.

[8] P. Stănică, T. Martinsen, S. Gangopadhyay, B. K. Singh, "Bent and generalized bent Boolean functions," Des. Codes Cryptogr., DOI 10.1007/s10623-012-9622-5.

[9] W. Su, A. Pott, X. Tang, "Characterization of negabent functions and construction of bent-negabent functions with maximum algebraic degree," arXiv: 1205.6568v1 [cs.IT], 30 May 2012. 